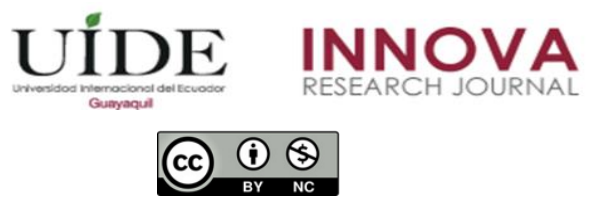

INNOVA Research Journal, ISSN 2477-9024

(Septiembre-Diciembre 2019). Vol. 4, No.3.2 pp. 80-88

DOI: https://doi.org/10.33890/innova.v4.n3.2.2019.1062

URL: http://revistas.uide.edu.ec/index.php/innova/index

Correo: innova@uide.edu.ec

\title{
Big Data, una herramienta para apoyar en decisiones del sector hotelero en Quito-Ecuador
}

\section{Big Data, a tool to avoid failures and support in decisions of the hotel sector in Quito-Ecuador}

\author{
Carlos Padilla Guevara \\ Universidad UTE, Ecuador
}

Autor para correspondencia: carlos.padilla@ute.edu.ec

Fecha de recepción: 12 de julio de 2019 - Fecha de aceptación: 18 de noviembre de 2019

\begin{abstract}
Resumen
Partiendo de un estudio basado en metodologías descriptiva-correlacional y observación, se concluyó que los hoteles de la ciudad de Quito no consideran a Big Data como una herramienta de apoyo para tomar decisiones. En el sector hotelero de la ciudad no se incursiona todavía con fuerza en el uso de esta nueva tecnología, debido al uso de herramientas más conocidas y tradicionales. Existe confusión entre lo que representan las herramientas cotidianas como bases de datos y el real potencial de contar con un Big Data. Los hoteles no lo perciben como algo que ya está funcionando realmente a nivel local; consideran que Big Data contiene gran cantidad de información más no calidad de esta. Sin embargo, les gustaría contar con más información que les sirva de apoyo para tomar las mejores decisiones. Contar con información clave, proveniente de diversas fuentes, que permita conocer a los clientes y su comportamiento, puede convertirse en el plus que la industria necesita para atraer nuevos consumidores. En el Ecuador. existen sectores productivos que ya están haciendo uso de esta tecnología; sin embargo, se evidencia la falta de personal capacitado, alta inversión que implica su implementación y falta de estructura de la información contenida.
\end{abstract}

Palabras claves: Big data; empresa; hotel; alojamiento; fracaso; Quito

\begin{abstract}
Based on a study based on descriptive-correlational methodologies and observation, it was concluded that the hotels in the city of Quito do not consider Big Data as a support tool for making decisions. In the hotel sector of the city, the use of this new technology is not yet strongly incurred, due to the use of more known and traditional tools. There is confusion between what everyday tools represent as databases and the real potential of having a Big Data. Hotels do not perceive it as something that is already really working locally; they believe that Big Data contains a great deal of information but not quality of it. However, they would like more information to help them make the best decisions. Having key information, from various sources, that allows to know customers and their behavior, can become the plus that the industry needs to attract new consumers. In Ecuador there are productive sectors that are already using this technology, however, there is a lack of trained personnel, high investment that implies its implementation and lack of structure of the information contained
\end{abstract}

Key words: Big data; company; hotel; accommodation; failure; Quito 


\section{Introducción}

A nivel mundial, cada vez se necesita contar con mejor calidad y cantidad de información, ya sea en el ámbito personal, profesional o corporativo. De tal manera que, a pesar de contar con herramientas como; nubes de información, redes sociales, grupos de profesionales, bibliotecas virtuales y distintas funcionalidades como: compartir e intercambiar información, realizar transacciones, relacionarse con nuevas personas, efectuar compras por internet, pagar los servicios básicos, comentar estados, jugar en línea, leer libros, escuchar música, mirar películas, encontrar estados de cuenta, reservas y comportamiento de mercados, aún no se potencializa la integración de estas herramientas.

Lo que se desea alcanzar con este estudio es determinar si las empresas hoteleras en Quito-Ecuador, están utilizando esta nueva tendencia para apoyarse en el momento que deben tomar decisiones o se han quedado con herramientas anteriores que seguramente han dado buenos resultados.

Las empresas y las personas que desean formar pequeñas y medianas empresas (pymes) o que se dedican a la industria del alojamiento en la ciudad de Quito, ¿se interesan en analizar la información disponible en estos espacios? ¿O será que ese tipo de información, no lo consideran relevante o necesitan algo distinto?

Es aquí donde nace un nuevo concepto llamado Big Data, por la necesidad de tener a la mano cualquier tipo de información que ayude a analizar, estructurar, construir, direccionar, perfeccionar alguna actividad o negocio en que las personas quieran involucrarse.

Está claro que las herramientas tecnológicas actuales, por sí solas, no tienen la capacidad de almacenar la gran cantidad de información que se genera constantemente, entonces, nace una de las definiciones más conocidas de Big Data: "Se trata de aquel conjunto de datos que, por su tamaño ingente, sobrepasa la capacidad de ser gestionado por bases de datos de integración tradicionales." (Martinez, 2015).

Ahora bien, ¿esta cantidad de información, contribuirá a disminuir el riesgo de fracasar al momento de emprender en alguna actividad llámese esta, una investigación, un emprendimiento, una planificación o un estudio detallado de un tema específico?

Considerando el concepto de fracaso: "Tener resultado adverso en un negocio" (Española, 2017), se concibe a esta herramienta como una alternativa que podría contribuir con más información que, aunque no garantice el éxito, brinde un panorama más amplio para analizar los pros y los contras de incursionar en algo nuevo y, en ciertas ocasiones desconocido.

Para pymes y cualquier tipo de organización, incluido el sector hotelero, contar con esta información y conocimiento de su utilización, podría representar una herramienta de gran valor al momento de tomar decisiones, es en el caso del hospedaje específicamente, en el que si se llegara a cruzar la información de comportamientos del consumidor, información de reservas, formas de pago, hábitos de viajes, alimentación, transporte. entre otras características, con sus datos personales como nacionalidad, periodicidad de viaje por tomar algunas variables, el 
empleo y uso de Big Data y herramientas de análisis de datos, arrojarían resultados muy útiles para tomar decisiones e impulsar estrategias que mejoren el funcionamiento de estos establecimientos.

El presente artículo es el primer paso para descubrir cómo los datos masivos, agrupados de una manera adecuada, pueden ser de gran ayuda para organizaciones de todo tamaño, que buscan ideas innovadoras y alternativas para evitar fracasos.

\section{Metodología}

En principio, las herramientas para determinar los resultados se basan en una metodología descriptiva-correlacional y observación, para esto, se ha tomado las siguientes variables: la empresa como tal en el mercado ecuatoriano, hoteles como caso de análisis, big data como herramienta de apoyo al momento de decidir y conocimiento sobre conceptos generales en el mundo empresarial como toma de decisiones y fracaso.

Los hoteles involucrados en este estudio son los cuarenta establecimientos de la ciudad de Quito que se encuentran en la categoría de cuatro estrellas y cinco estrellas.

$\mathrm{Al}$ obtener un cruce de datos entre estas variables, los establecimientos en análisis y los datos obtenidos tanto de los clientes como de sus comportamientos, se podría llegar a contar con información de calidad que realmente influya al momento de decidir.

\section{Desarrollo}

\section{Realidad del Big Data}

Big Data es lo que muchos consideran el siguiente paso o concepto a convertirse en tendencia. En el World Economic Forum, se habla de tres pasos para armar esta estructura: "El primer paso consiste en recoger información de los consumidores y obtener los Big Data; el segundo, en escuchar sus opiniones; y el tercero, en aprovechar el canal para ofrecerles productos y servicios directamente y mercadear con ellos" (World Economic Forum , 2018).

Si se parte de este criterio para armar un Big Data, lo primero que se debe considerar es la información que genera cada individuo u organización en su día a día; identificar qué se busca de esta información, y hacer visibles las distintas formas de utilizarla para convertirla en información útil.

Hoy en día, a pesar de estar en una era donde la tecnología evoluciona constantemente, la necesidad de contar con información que genere confianza y sobre todo, sea de ayuda al momento de tomar decisiones, aún cuenta con bloqueos principalmente sociales; debido a características propias de las distintas generaciones. Es el caso de la generación silenciosa (nacidos entre 1920 y 1940) y de la generación llamada baby boomers (nacidos entre 1946 y 1965), quienes no están dispuestos a perder su intimidad; por lo tanto, si se sabe que toda acción cotidiana deja huellas y que esas huellas o señales son base fundamental en la era digital, ¿cómo hacer que esa parte de la sociedad, que sigue siendo significativa, utilice su teléfono móvil ya sea 
para chatear, ingresar a redes sociales, genere y reciba correos electrónicos, realice transacciones bancarias, pague sus servicios básicos, lea libros, realice reservas y compras en línea, entre otras actividades que actualmente permiten estos dispositivos?

Toda empresa u organización, debe analizar en la actualidad, aspectos que anteriormente eran considerados de menor importancia y comenzar a prestarles más atención al momento de manejar nuevas variables como hábitos de compra, reconocimiento del valor que los clientes le dan a los productos, servicios, nuevas ideas, descubrimiento de nuevas necesidades, mejora y reestructuración de sus diseños de organización junto con la toma de decisiones.

Ya no es suficiente, contar con un almacén de datos (data warehouse), pues la mayor diferencia entre este y un Big Data, radica en la evolución de las tecnologías, rapidez de obtención de información y reducción de costos (Valls, 2017).

La necesidad de ser más competitivo y contar con una aceleración concerniente a innovación, provocan una personalización en cada uno de los productos y servicios actuales, priorizando el criterio y la necesidad del cliente final antes que la opinión generada desde el interior de la empresa, de tal foma que se evidencian mejoras en la toma de decisiones, acciones en tiempo real y optimización en el proceso de aprendizaje en la gestión de relacionarse con los clientes (Mckinsey Global, 2016). Entre las empresas más conocidas que han implementado estos elementos como parte de su reorganización a nivel mundial, son: Google, Alibaba, Amazon, Microsoft, Facebook; lo que lleva a determinar que los Big Data también son: "activos de información de gran volumen, alta velocidad y gran variedad que demandan soluciones eficientes e innovadoras de procesar la información que permiten aumentar los conocimientos, la toma de decisiones y la automatización de procesos" (Calle, 2017).

Este procesamiento de información es realizado con tecnología dedicada al análisis de grandes cantidades de información, como por ejemplo Hadoop que no es más que una aplicación de código abierto (open source) capaz de procesar grandes cantidades de datos (Dominguez A., 2014).

En otras palabras, Hadoop es un sistema de archivos que los almacena de forma distribuida, es decir, en lugar de guardar íntegramente un archivo y replicarlo en varias unidades, reparte el archivo en varias unidades y así optimiza espacios de almacenamiento (Galimany, 2014).

\section{Big Data y la empresa ecuatoriana}

El Instituto Nacional de Estadísticas y Censos (INEC), con datos ingresados y analizados hasta el año 2012,indica que en Ecuador se encuentran registradas 704.556 compañías; Pichincha, con 151.671 empresas, es la provincia con mayor número de registros $\mathrm{y}$, dentro de esta, sólo la ciudad de Quito abarca el $47 \%$ del total provincial, lo que representa el 19\% a nivel nacional.

Dentro de estas cifras, los pequeños emprendimientos son los que predominan a nivel nacional con 89,6\%. Las pequeñas empresas completan la participación con el 8,2\%; las 
medianas empresas, con $1,7 \%$, y las grandes empresas, con $0,5 \%$, como muestra la tabla 1 . (INEC, 2018)

\section{Tabla 1}

Participación empresas Quito

\begin{tabular}{llll}
\hline Empresas Nivel Nacional & Pichincha & \multicolumn{2}{c}{ Quito } \\
\hline 704556 & 151671 & 71285,37 & $47 \%$ \\
Microempresas & Pequeña Empresa & Mediana empresa & Grandes Empresas \\
$89,60 \%$ & $8,20 \%$ & $1,70 \%$ & $0,50 \%$ \\
\hline
\end{tabular}

Adaptado de: Instituto Nacional de Estadísticas y Censos (2018)

Para el año 2018, se tiene una base de 843.745 empresas registradas según el INEC, las que no han sido analizadas aún para determinar la participación de acuerdo al tipo de empresa.

Se han realizado estudios en varios sectores productivos del país. La línea farmacéutica es una de las que más llama la atención; principalmente, una cadena a nivel nacional cuyo cruce de información, entre los datos de sus tarjetas de afiliación y los registros de ventas anclados a estas tarjetas, ha arrojado datos interesantes sobre tendencias de compra de los clientes (El Comercio, 2017). A pesar de esto, otros sectores productivos no se deciden aún por esta tendencia debido a dos factores: el primero, los costos elevados al momento de segmentar información y el segunda los pocos expertos existentes en el mercado ecuatoriano.

Otro de los sectores productivos, es el de alojamiento en la ciudad de Quito; tomando como referencia al Reglamento de Alojamiento Turístico, avalado por el Ministerio de Turismo del Ecuador ente regulador de este sector, los establecimientos de alojamiento considerados para el presente análisis, son los hoteles, que entran en la categoría Hotel, cuya nomenclatura es la letra $\mathrm{H}$.

Un hotel es considerado como un establecimiento de alojamiento que cuenta con instalaciones privadas con baño privado, que ocupa un edificio completo o una parte independiente de este, y que brinda servicio de alimentos y bebidas ya sea en un restaurant propio o cafetería.

Las categorías asignadas en las que se puede ubicar un hotel, van desde dos hasta cinco estrellas, siendo una de las características que deben cumplir los establecimientos para posicionarse, dentro de la categoría de cuatro estrellas es contar con el $20 \%$ de su personal capacitado profesionalmente o certificado en competencias laborales; mientras que, para estar considerado como un hotel de cinco estrellas, se debe contar con el $30 \%$ de su personal capacitado o certificado profesionalmente. (Mintur, 2016).

En hoteles de cuatro estrellas, las prescripciones se refieren a que: el personal hable al menos una lengua extranjera, debe representar como mínimo el 15\% de su nómina; mientras que, en hoteles de cinco estrellas, el personal mínimo que debe dominar una lengua extranjera debe representar al 25\% del total de colaboradores (Mintur, 2016) 
Según el catastro de hoteles de la ciudad de Quito, el número de establecimientos se muestra en la tabla 2.

Tabla 2.

Participación Hoteles en Quito

\begin{tabular}{lll}
\hline Clasificacion & Categoria & Cantidad Establecimientos \\
\hline Hotel & Dos Estrellas & 25 \\
Hotel & Tres Estrellas & 52 \\
Hotel & Cuatro Estrellas & 29 \\
Hotel & Cinco Estrellas & 11 \\
\hline
\end{tabular}

Adaptado de: Catastro de Establecimientos de alojamiento (2018)

Considerando este número de entidades, indistintamente de las posibilidades económicas, no se ha encontrado un real interés en el uso de Big Data a nivel local, a pesar de contar con suficientes variables que resultarían interesantes de analizar, dentro de este giro de negocio como pueden ser, datos obtenidos al momento de la reserva, gustos de los turistas, tendencias de consumo, preferencias en alimentación, información al momento del registro, comentarios sobre el servicio, forma de pago, lugar de procedencia y preferencias de viaje.

A estas variables también se las puede clasificar según el tipo de cliente que se hospeda y según el uso que cada uno le da a la tecnología. Es así, que se puede hablar de turistas de primera generación, quienes prefieren contratar todo por medio de una agencia de viajes; los de segunda generación, son los que buscan destinos en internet, consiguen la información que necesitan, pero ocasionalmente contratan los servicios por esa vía, y los llamados de tercera generación, conocidos porque todo lo hacen conectados a internet, principalmente desde su móvil y no se enfocan únicamente en buscar información sino en planificar y dejar listo todo lo que concierne a la organización de su viaje (Maldonado J., 2015).

De acuerdo al Instituto Turístico y Hotelero de México, se asegura que las nuevas tendencias que enfrentará el sector hotelero son las siguientes: incremento en el uso de internet, evolución de la demanda, aumento de la oferta acompañada de diferenciación, conocimiento del comportamiento del consumidor, competencia con otros tipos de hospedajes, acceso a nuevas tecnologías sin causar afectación al medio ambiente y preocupación por la profesionalización del capital humano (Maldonado J., 2015).

Es a partir de esta información, que la persona, empresa, grupo corporativo o cualquier tipo de organización que decida dedicarse al alojamiento, debe considerar muy seriamente los constantes cambios que se presentan en los nuevos turistas, que no se conforman con conocer nuevos lugares sino que requieren llenar sus expectativas a nivel personal, familiar y grupal, hospedándose en sitios que les brinden más comodidad, más servicios, más seguridad, más atención en los detalles, que se adapten y que realmente conozcan lo que sus visitantes buscan, sin dejar de lado el acompañamiento tecnológico. 
En Ecuador, especificamente en la ciudad de Quito, tomando como referencia el sector hotelero, se evidencia, por un lado, desconocimiento sobre lo que es Big Data, lo que representa y lo que se puede obtener a partir de la utilización de esta tecnología y, por otro lado, falta de interés y resistencia a la utilización de Big Data desde el momento de la puesta en marcha de la empresa hasta el día a día de sus operaciones mientras no se observe un crecimiento en su aplicación real dentro del medio.

Se verifica que, efectivamente, hay instituciones del sector hotelero que sí manejan bases de datos y que éstas, en muchos casos, son utilizadas para tomar decisiones en cuanto a estrategias comerciales, promociones, diseño de nuevos productos, análisis de porcentajes de ocupación, rotación, manejo de inventarios, mantenimientos. Sin embargo, los datos no son visualizados en su real potencial como para analizar tendencias de consumos, gustos de los visitantes, fidelización de clientes.

\section{Dificultades de Big Data}

Una de las dificultades, con respecto al uso de Big Data, es que los datos no tienen una estructura estandarizada, sino que dependen del criterio de quien la deposita y la forma como la tiene organizada. Por esta razón, a los datos almacenados se los conoce como estructurados y no estructurados, tal como muestra la figura 1.

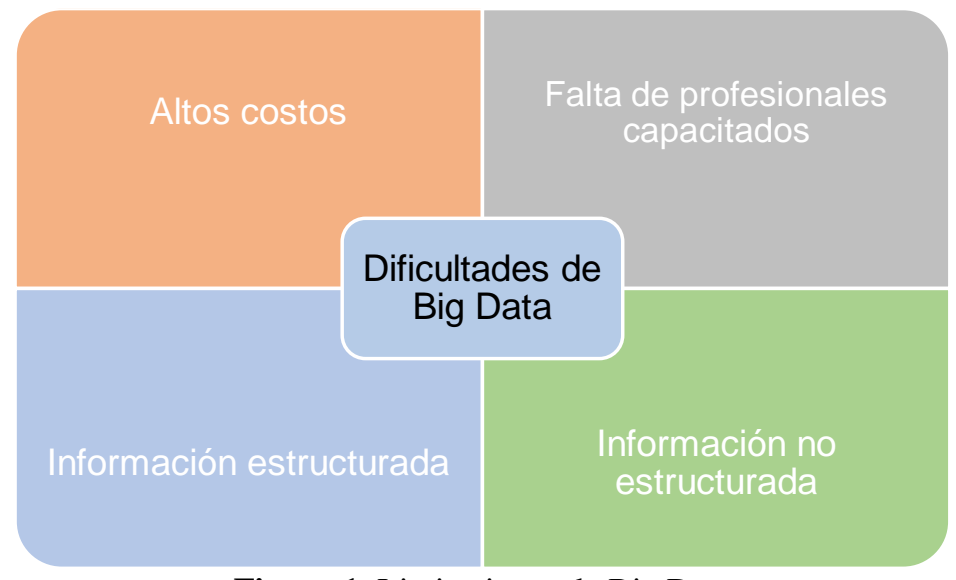

Figura 1. Limitaciones de Big Data

Elaborado por autores

No solo la falta de una estructura es un limitante; al ser una nueva tendencia, no se encuentra aún en el mercado laboral un número considerable de profesionales que sean expertos en esta tecnología por lo que los costos de algún desarrollo sigue siendo considerablemente alto, principalmente si se acude a un experto externo.

El uso de esta nueva tecnología permite rediseñar ideas de negocios, analizar su organización interna, mejorar sus procesos y pensar más en el cliente final que en lo que la organización le ofrece (Evans, 2014). Es así que, aunque el criterio del cliente es muy importante, ya no prevalece lo que se le pueda ofrecer sino más bien, se busca cubrir las necesidades, mediante un análisis a fondo de su comportamiento y de los diferentes estilos de vida para responder a la pregunta: ¿Qué tipo de comprador es? 
Comprador de valor.- Son aquellos que se fijan en marcas de prestigio, que les den cierto estatus, puede ser que hasta se identifiquen con la marca que satisface todas sus necesidades. No están interesados en nuevas marcas en la que seleccionaron, cubre todas sus expectativas. (Valls, 2017).

Compradores híbridos.- No les interesa ser fieles a una marca, sino que pueden comprar tanto lo que sea caro como lo que sea barato, mientras cumpla sus expectativas, considerará que está bien. (Valls, 2017).

Compradores racionales.- El equilibrio debe estar dado por la calidad y el precio de lo que están adquiriendo, no importa si es caro o barato, siempre y cuando la calidad y las garantías que se le ofrece, sea lo que necesita. Planifican sus compras y colocan entre sus gastos principales, la cultura y la alimentación. Si quieren regalar algo, se fijarán en la utilidad antes que en otro factor (Teinteresa, 2018)

Comprador por precios.- No se fija si será bueno o malo, duradero o no, el principal parámetro para estas personas es el precio, lo que adquieren debe tener un precio bajo. (Valls, 2017)

Por esta razon, se convierte en una tarea de las organizaciones, el buscar la manera y las herramientas al momento de atender adecuadamente a sus clientes, desarrollando productos y servicios flexibles, de tal manera, que sus potenciales y actuales consumidores, encuentren en su oferta lo que están buscando.

\section{Conclusiones}

El crecimiento de esta nueva tendencia llamada Big Data, se evidencia en que constantemente se va incrementando el número de especialistas y profesionales dedicados al análisis de datos, y es que es tal, la cantidad de información que se genera a diario principalmente proveniente de dispositivos electrónicos, que en un futuro muy cercano, no será descabellado imaginar que todo negocio, incluidos los del sector de alojamiento, utilicen estas herramientas para apoyarse en la toma de decisiones.

Cada vez que se utiliza tecnología, se debe considerar que las acciones realizadas dejan una marca, una señal o una huella de lo ejecutado. Hoy en día, a pesar de tener libre acceso a herramientas tecnológicas, aun se puede identificar cierta resistencia a su uso, principalmente por parte de generaciones anteriores, cuya formación fue muy distinta a la impartida en la actualidad, uno de los casos más relvantes en la Industria de la Hospitalidad es Airbnb la plataforma que viabiliza la accesibilidad a hospedaje en varios países del mundo permitiendo al turista la accesibilidad a diferentes ofertas de bienes y servicios.

La manera de administrar las empresas de alojamiento como sectores proveedores de una capacidad inmobiliaria, que debe romper ciertos paradigmas, enfocarse más en la necesidad del cliente en cuanto a producto, servicio, precio, canales de distribución y accesibilidad a la promoción que ofrecen, permita analizar variables nuevas como comportamiento del 
consumidor, hábitos de consumo, entre otras, cuya finalidad es satisfacer las necesidades del cliente aunque esto, conlleve una reestructuración corporativa.

En el Ecuador a nivel empresarial, específicamente en el sector hotelero, el uso de Big Data es mínimo en comparación al volumen de información que se puede generar y a la diversidad de variables que se podrían analizar. Esto ha llevado a no mostrar un real interés en el uso de nuevas tecnologías y mantener el uso de herramientas más tradicionales como bases de datos, desaprovechando lo que representaría un detalle y análisis más profundo de la información que facilitaría la satisfacción de necesidades del turista extranjero y nacional que frecuenta el país y que genera ingresos en el sector turístico.

Para poder potenciar la información generada día a día, Big Data se muestra como la tendencia de futuro en el corto, mediano y largo plazo, para esto, es importante dar el primer paso y entender al cliente desde todos los ángulos, Big Data no fue creado para un sector productivo específico, no tiene un solo sector en el cual se pueda aplicar, lo interesante de su uso es la finalidad que se le vaya a dar a los datos arrojados.

\section{Bibliografía}

Acerca de la aplicación de MapReduce + Hadoop en el tratamiento de Big Data. (s.f.).

Calle, J. (2017). Revolución Big Data en el turismo. Análisis de las nuevas fuentes de datos para la creación de conocimiento en los Destinos Patrimonio de la Humanidad de España.

Comercio, E. (19 de mayo de 2017). El big data le haxe un zoom al consumidor. El Comercio.

Dominguez A., Y. A. (2014). Acerca de la aplicación de MapReduce + Hadoop en el tratamiento de Big Data. La Habana: Revista Cubana de Ciencias Informáticas.

Española, R. A. (2017). Diccionario de la lengua española.

Evans, P. (2014). De la deconstrucción a los Big Data. BBVA.

Forum, 2. W. (2018). El Foro Económico Mundial. Obtenido de El Big Data y los consumidores | Foro Económico Mundial: https://es. weforum.org/agenda/2017/12/el-big-data-y-losconsumidores

Galimany. (2014). La creación de valor en las empresas a través del Big Data. 33.

Gartner. (2016). IT Glossary. Obtenido de https://www.gartner.com/it-glossary/big-data.

INEC. (2018). Empresas de Ecuador: Quito lidera en cantidad. Obtenido de elemprendedor.ec: http://www.elemprendedor.ec/quito-lider-empresas-de-ecuador/

Maldonado J., S. S. (2015). El Big Data como ventaja competitiva. Querétaro, México: Universidad Autónoma de Querétaro.

Martinez, J. M. (2015). "Big Data"; aplicación y utilidad para el sistema sanitario. Farmacia Hospitalaria, ISSN: 2171-8695., 1.

Mckinsey Global, I. (2016). The Age of Analytics: Competing in a Data-Driven World.

Mintur. (2016). Reglamento de Alojamiento Turístico. Quito: Ministerio de Turismo.

Teinteresa. (2018). Qué tipo de comprador eres? Obtenido de teinteresa.es: http://www.teinteresa.es/espana/tipo-comprador_0_1049295310.html

Valls, J. F. (2017). Big Data, Atrapando al consumidor. Barcelona, España: Profit. 